Retos, $n^{\circ} \mid 4$, vol.VIII, 2017

\title{
Nivel de uso de las redes sociales en el proceso de comunicación en las MIPES de Cuenca
}

\section{Level of use of social networks in the communication process in the micro and small sized enterprises of Cuenca}

Efrén Aucay Piedra es ingeniero commercial por la Universidad Politécnica Salesiana (Ecuador) (iaucay@est.ups.edu.ec) (http://orcid.org/0000-0003-3617-5235)

Priscilla Herrera Torres es profesora/investigadora de la Universidad Politécnica Salesiana (Ecuador) (dherrerat@ups.edu.ec) (http://orcid.org/0000-0002-0693-2621)

\begin{abstract}
Resumen
La investigación se desarrolló en Cuenca, tercera ciudad más importante del Ecuador, cuyo objetivo principal se enfocó en analizar el nivel de uso, así como la administración de redes sociales y aplicaciones móviles en los procesos de comunicación y mercadotecnia en las Micro y Pequeñas Empresas (MIPES). Debido a que la globalización del internet ha hecho que las empresas deban adaptarse a entornos cada vez más complejos y competitivos, creando estrategias de valor para llegar a satisfacer a un nuevo estilo de demandantes, que influenciados por publicaciones en diferentes medios virtuales, están cada vez más informados, lo que repercute en una sociedad más exigente de confort. La investigación es de tipo cuantitativa, la muestra se tomó de las Micro y Pequeñas empresas de la ciudad. Para la recolección de información se implementó una encuesta, instrumento que fue aplicado a los establecimientos seleccionados. Los constructos de medición se validaron mediante el uso del alfa de Cronbach. Los resultados obtenidos demuestran un bajo nivel de adopción de redes sociales en la microempresa y un nivel medio en la pequeña empresa. Adicionalmente, se observa que en los establecimientos que utilizan las redes sociales y las aplicaciones móviles para actividades de comunicación y mercadotecnia, éstas no se explotan en su totalidad por múltiples factores como: desconocimiento en el uso y administración, no lo consideran necesario, falta de confianza y credibilidad entre otros aspectos.
\end{abstract}

\begin{abstract}
The research was carried out in Cuenca, the third most important city of Ecuador. Its main objective is to analyze the level of use, the administration of social networks and mobile applications, in the communication and marketing processes in Micro and Small sized enterprises (MIPES). Due to the globalization of the Internet, it has meant that companies have to adapt to increasingly complex and competitive environments, creating value strategies to meet a new style of demanders, influenced by publications in different virtual media. Consumers are increasingly informed, and it has repercussions in a more demanding society of comfort. It is a quantitative research, and the sample was taken from the Micro and Small enterprises of the city. For the collection of information, a survey was implemented. Then, an instrument was applied to selected establishments. The measurement constructs were validated using the Cronbach's alpha. The results show a low level of adoption of social networks in the microenterprise and a medium level in the small company. Additionally, it has been observed that establishments that use social networks and mobile applications for communication and marketing activities, it is not fully exploited, due to many factors such as: the lack of knowledge about the use and administration of social networks, it is deemed necessary, and the lack of trust and credibility among others.
\end{abstract}

\section{Palabras clave | keywords}

Redes sociales, aplicaciones móviles, comunicación, marketing, microempresas y pequeña empresas. Social networks, mobile apps, communication, marketing, micro and small sized enterprises. 


\section{Introducción}

Las empresas del siglo XXI siguen transformándose por la globalización del internet y el crecimiento vertiginoso de las redes sociales y aplicaciones móviles, hecho que ha ocasionado cambios en los ámbitos interno y externo de las empresas, las mismas que deben adaptarse a entornos que cada día son más complejos y competitivos, donde los administradores deben poseer habilidad y experticia para crear estrategias de valor y para llegar a satisfacer a un nuevo estilo de demandantes, que influenciados por las publicaciones presentadas a través de los diferentes medios virtuales, están cada vez más informados; lo que repercute en una sociedad más exigente de confort. Las redes sociales y aplicaciones móviles han llegado para quedarse y constituirse en un aliado clave para la gestión empresarial en los campos de comunicación y mercadotecnia. Por tal razón amerita investigar el papel que desempeñan las redes sociales y aplicaciones móviles, empleadas en actividades de comunicación y mercadotecnia en las MIPES de la ciudad de Cuenca, en ámbitos de cobertura, nivel de uso, administración y aspectos de percepción.

\subsection{Las Micro y Pequeñas Empresas (MIPES)}

Las Micro y Pequeñas Empresas (MIPES) son establecimientos que tienen ciertas características en común a nivel mundial, pero existen algunas particularidades a nivel de cada territorio. Como se citó en Tobar (2015, p.81) "La Unión Europea clasifica como microempresa a toda unidad económica con menos de diez trabajadores, así como aquellas que tienen un nivel de ventas anuales menor a dos millones de euros". En Chile, de acuerdo a Dini y Stumpo se clasifican como "microempresas aquellas unidades productivas que ocupan entre 1 y 4 personas, y pequeñas empresas a las que ocupan entre 5 y 49 personas" (2002, p.10).

En Ecuador, según el directorio de empresas y establecimientos (INEC, 2014), se clasifica a las MIPES por el personal laboral empleado y nivel de ingresos anuales, denominando como microempresas aquellos establecimientos que tienen de 1 a 9 empleados y ventas anuales menores o igual a $\$ 100000$. En cuanto a las pequeñas empresas, por tener de $10 \mathrm{a}$ 49 empleados e ingresos por ventas anuales de \$100001 a un 1000000 . Por otra parte, la naturaleza y razón de ser de las MIPES es de carácter sencilla y autónoma, acorde con Anzola es por "la necesidad de las personas de ser independientes en el aspecto laboral y económico" (2010a, p.14). 
En este sentido las MIPES desde sus inicios han sido creadas y administradas en su mayoría por sus propietarios, personas innovadoras y creativas que aportan al crecimiento económico y al desarrollo de los pueblos, llegando a constituirse en los negocios más representativos en América Latina. Según Anzola (op. cit) "la pequeña empresa junto con la micro empresa representan el 95\% de los negocios en América Latina" (2010b, p.17). Sin embargo, la globalización del internet se ha convertido en un desafío para algunos establecimientos. Según Liberona y Ruiz (2013, p.152) "se está desarrollando una red cada vez más compleja de relaciones de conocimiento dentro y fuera de las fronteras organizacionales". No obstante, internet y la telefonía móvil, según Muñiz y O’Guinn como se citó en Casaló, Flavián \& Guinalíu (2012, p.43) "ha permitido la constante evolución de las redes sociales gracias a la posibilidad que ofrecen dichos canales para unir individuos que se encuentran geográficamente dispersos pero que tienen un interés común".

El internet y la telefonía móvil han permitido el surgimiento y proliferación de las redes sociales y aplicaciones móviles, los mismos que están al alcance de los individuos y las empresas; medios que sirven para la gestión de las relaciones con todos los involucrados en algún tema de interés en común, que acorde a Mitchell como se citó en Lozares (s/f, p.108) "pueden definirse como un conjunto bien delimitado de actores -individuos, grupos, organizaciones, comunidades, sociedades globales, etc.- vinculados unos a otros a través de una relación o un conjunto de relaciones sociales". En este mismo sentido, las redes sociales nos permiten estar a seis pasos de interactuar con las personas a nivel global, siguiendo a González (2014, p. 279) quien indica que "todos estamos conectados con cualquier otra persona del planeta a través de una cadena de conocidos, que no tiene más de cinco intermediarios".

En el ámbito comunicativo en las empresas las redes sociales y aplicaciones móviles, según Richard y Chandra como se citó en Bigné, Küster y Hernández (2013, pp. 9-10) han:

(...) provocado profundos cambios en la forma en que las empresas se contactan con sus clientes posibilitando, por una parte, una comunicación selectiva y una posición más activa del receptor dando al consumidor un mayor poder sobre el proceso de comunicación en comparación con los medios tradicionales y, por otra parte, facilitando el modo en que los consumidores y usuarios se relacionan entre sí. 
En este sentido las redes sociales y las aplicaciones móviles en las empresas están "intentando tratar de ser otro contacto con los que frecuentemente mantienen una relación, permitiendo así esta comunicación bidireccional" (Romero, Alarcón y Gómez, 2011, p. 201). Ante estos aspectos, los servicios de las redes sociales y aplicaciones móviles en la esfera de la comunicación, de acuerdo a Barker, Barker, Bormann y Niher "simplifican el proceso de intercambio de información" (2015, p.178). Los mismos que son necesarios para una comunicación efectiva, que acorde a Garrido la empresa en estos tiempos "requiere una gestión de comunicación eficiente, rentable y sin detalles dejados al azar" (2004, p. 22). En este mismo orden de ideas Lam, Hair y McDaniel explican que "las empresas están interesadas en la comunicación en línea porque goza de enorme popularidad" (2014, p. 308). No obstante

las PYMES tienen muchos problemas para administrar los escasos recursos de comunicación que tienen para sus empresas, ya sea porque gastan en campañas que no son las adecuadas o porque van dirigidas no siempre al público apropiado desperdiciando tiempo y dinero (Hoyos y Lasso, 2017, p. 67).

En el campo de la mercadotecnia, las redes sociales se presentan como un pilar fundamental para brindar información de campañas publicitarias y promocionales. Según Barker (2015) en su misma publicación manifiesta que las redes sociales pueden ser una alternativa para localizar clientes potenciales y hacer llegar alguna promoción. Estos medios facilitan el marketing social, acorde a Laudon y Guercio "El marketing social implica el uso de las redes sociales y comunidades en línea para construir marcas y aumentar los ingresos por ventas" (2014, p.175).

En este mismo contexto, aparece el término marketing móvil, que consiste en:

(...) el uso de los smartphones, también llamados teléfonos inteligentes, que no solo han cambiado nuestra vida, más en contacto con la tecnología, sino también la forma en que consumimos contenidos y accedemos a la información. De este modo ha surgido una nueva forma de entender el marketing, basada en el uso de los teléfonos móviles para transmitir mensajes, servicios e ideas promocionales... a pesar de ser una disciplina joven se diferencia del marketing tradicional en disponer una mayor variedad de medios o herramientas para llegar a los usuarios (Estrade et al., 2013, p. 37). 
Sin embargo existen dificultades para medir las actividades de mercadotecnia dirigidas a través de las redes sociales digitales (RSD), según Clemons como se citó en Uribe, Rialp y Llonch (2013, p. 208) "hay un consenso entre los profesionales del marketing en que existe un problema complejo a la hora de medir y monetizar las actividades de marketing en las RSD".

Las redes sociales y las aplicaciones móviles se están constituyendo con rapidez en una herramienta para compartir ideas, entre todos los stakeholders en el ámbito corporativo; además "es una importante ventaja competitiva no solo en términos de nuevos mercados, sino en términos de Know-How" (González, 2014, p. 52). Así, el uso y administración de las redes sociales y aplicaciones móviles en las empresas comienza por el alto nivel de acogida de estos por la sociedad en general, creciendo a un ritmo constante desde el año 2005 (Barker, 2015), sin ningún indicio de disminución.

En este escenario las redes sociales para la empresa según la conectividad, acorde a Ureña y Valenzuela "representan potenciales repositorios de información” (2011, pp. 131, 132). Ante estas circunstancias, los negocios están expuestos a este nuevo sistema de comunicación, pues según Valls (2016, p. 24), la empresa "requiere de estrategias comunicativas nuevas que aprecien estos cambios sociales, se adapten al nuevo entorno y le den más importancia a la comunicación”, aunque es preciso hacer hincapié en los diferentes tipos de redes sociales que tienen mayor impacto en las empresas y la sociedad, las mismas que se ilustran en la tabla 1, incluyendo el criterio de algunos autores al respecto.

Tabla 1. Tipos de redes sociales

\begin{tabular}{|l|l|l|}
\hline Red social & \multicolumn{1}{|c}{ Tipo de red social } & \multicolumn{1}{c|}{ Al respecto los autores expresan: } \\
\hline Facebook & $\begin{array}{l}\text { Red social generalista; Puesto } \\
\text { que albergan a todo tipo de } \\
\text { personas y admite todo tipo de } \\
\text { contenido (Martos, 2010, p.73) }\end{array}$ & $\begin{array}{l}\text { "Las páginas profesionales de } \\
\text { Facebook páginas de fans o fan page } \\
\text { permite a las empresas y profesionales } \\
\text { tener una presencia en Facebook que } \\
\text { es visible, no solo por los usuarios de } \\
\text { Facebook, sino por cualquier usuario } \\
\text { de internet" (Carballar, 2012, p. 93) }\end{array}$ \\
\hline YouTube & $\begin{array}{l}\text { Es una red social de contenidos, } \\
\text { donde el principal factor es el } \\
\text { material que en esa red se com- } \\
\text { parte (Junta de castilla y León, } \\
\text { 2012, p.8) }\end{array}$ & $\begin{array}{l}\text { "Utilizar YouTube en las estrategia } \\
\text { de redes sociales de su empresa, } \\
\text { le permite diferenciarse de sus } \\
\text { competencia" (Mejía, 2013a, p.142) }\end{array}$ \\
\hline
\end{tabular}




\begin{tabular}{|c|c|c|}
\hline Red social & Tipo de red social & Al respecto los autores expresan: \\
\hline Twitter & $\begin{array}{l}\text { Es una red social con un servicio } \\
\text { de microblogging gratuito que } \\
\text { permite a los usuarios enviar y } \\
\text { compartir entradas cortas, de- } \\
\text { nominadas "tweets", no mayores } \\
\text { de } 140 \text { caracteres. (Medina y } \\
\text { Montjoy, 2015, p. 8) }\end{array}$ & $\begin{array}{l}\text { "Esta red social se ha convertido en } \\
\text { un medio social de interés para que } \\
\text { las empresas puedan comunicar } \\
\text { determinadas ofertas, resolver las } \\
\text { dudas..." (Gómez \& Suárez, 2012a, } \\
\text { p.195) }\end{array}$ \\
\hline LinkedIn & $\begin{array}{l}\text { Es una red social profesional } \\
\text { (Hütt, 2012, p.123) }\end{array}$ & $\begin{array}{l}\text { Linkedln, "se ha convertido en un } \\
\text { soporte especialmente atractivo } \\
\text { para la inclusión de anuncios y } \\
\text { mensajes publicitarios que se } \\
\text { pueden dirigir a un público objetivo } \\
\text { muy bien definido" (Gómez \& } \\
\text { Suárez, 2012b, p. 200) }\end{array}$ \\
\hline Instagram & $\begin{array}{l}\text { Es una red social basada en } \\
\text { compartir elementos multime- } \\
\text { dia (Estrade, Soro \& Hernán- } \\
\text { dez, 2013, p.131) }\end{array}$ & $\begin{array}{l}\text { En Instagram, "Las imágenes para } \\
\text { compartir se pueden modificar a } \\
\text { través de una serie de filtros que se } \\
\text { han ido sofisticando a lo largo de } \\
\text { las sucesivas versiones del progra- } \\
\text { ma" (Mejía, 2013d, p.152) }\end{array}$ \\
\hline
\end{tabular}

En este contexto es preciso destacar que Facebook en el Ecuador "cuenta con una audiencia de 8900000 de usuarios, de los cuales 1400000 son administradores de páginas Facebook, muchas de las cuales pertenecen a marcas, emprendimientos" (Formación gerencial, 2015). Por otro lado en relación a las aplicaciones móviles, Barker et al. (2015b, p. 263) manifiestan que "la mayoría de las personas tienen dispositivos móviles a la mano, de modo que están conectadas a las redes sociales las 24 horas al día los siete días a la semana". En palabras de Estrade et al. (2013, p. 129):

(...) las redes sociales y los dispositivos móviles de última generación han establecido una interesante relación de simbiosis. Unos (las redes sociales) han ofrecido un medio que permite a las personas comunicarse de una forma más efectiva, hasta el punto de que compartir información con otra persona mediante tu móvil se puede convertir en una experiencia placentera. Los otros los dispositivos móviles son el aquí y ahora la posibilidad de poder interactuar con tu comunidad virtual.

Asimismo se hace referencia a los servicios ofrecidos tanto de datos como de voz en el mismo dispositivo. De acuerdo a Laudon (2012, p. 264): 
la mensajería instantánea es un tipo de servicio de chat que permite a los participantes crear sus propios canales privados. El sistema de mensajería instantánea alerta al usuario cada vez que alguien en su lista privada está en línea, de modo que pueda iniciar una sesión de chat con otros individuos. Algunos de los sistemas de mensajería instantánea para los consumidores son Yahoo!, Messenger, Google Talk, Windows live Messenger.

Las aplicaciones móviles nativas para los dispositivos "deben ser descargadas de una tienda de aplicaciones (App Store de Apple, Google Play de Android, Windows Mobile Marketplace para Windows Phone, etc." (Estrade et al., 2013, p. 92). En este contexto y haciendo hincapié en los dispositivos móviles en Ecuador, según Espinoza como se citó en Rosero \& Montalvo (2015), uno de los mercados que crece dinámicamente son los de los smartphones, que hasta octubre de 2014 superaron los 18 millones en ventas, donde 3 de cada 5 son teléfonos inteligentes. De acuerdo con estos planteamientos, las redes sociales y aplicaciones móviles son cada vez más amplias y versátiles, las mismas que están al alcance de todo tipo de empresa, independientemente del sector económico e industria a la que pertenezca.

En tal virtud en la empresa el "conocimiento existente tiene que aplicarse y el nuevo debe generarse para satisfacer las necesidades de los clientes internos y externos" (Jiménez, 2011, p.175). Las redes sociales y aplicaciones móviles, son un aliado clave, a la vez que una ventana hacia la eficiencia. Si los esfuerzos de administración son dirigidos a la audiencia indicada y el momento oportuno, se puede lograr ubicar las MIPES en una mejor posición competitiva en el mercado. De acuerdo con Moyano, Bruque \& Eisenberg (2007, p.162) "las organizaciones deberían considerar a las redes sociales como otro activo empresarial más, o como una dimensión de capital humano que merece atención".

Por último, es necesario hacer referencia a la cifra de MIPES en Ecuador. De acuerdo al directorio de empresas y establecimientos (INEC, 2014) el país registra 843644 empresas, de las cuales el 97\% de los establecimientos están dentro de las categorías MIPES. En concordancia con esta información, en las cuatro provincias más pobladas del Ecuador es donde se concentra el $57 \%$ de empresas, a saber: en la provincia de Pichincha se asientan el 23\% de establecimientos, en Guayas el 19\%, en Manabí el 8,3\% y en la provincia del Azuay el 5,8\%. 
En la ciudad de Cuenca, capital de la provincia del Azuay y tercera ciudad más importante del país -objeto del presente análisis-, es donde se enfoca la investigación, de acuerdo a la información proporcionada por el Ministerio de Industrias y Productividad (MIPRO). El 96\% de establecimientos están categorizados como MIPES.

En base a esta información se demuestra que son negocios altamente representativos de la ciudad ut supra reseñada. No obstante, según Elizalde y Morocho (2014, p. 45) "las PYMES cuencanas no dan el suficiente valor e importancia a las redes sociales, por desconocimiento de uso de las mismas o por desconfianza de efectividad".

De acuerdo con estos planteamientos, se formulan las variables de estudio, partiendo por examinar la situación actual de las empresas en relación a: i) nivel de uso; ii) gestión; iii) conocimiento para administrar las redes sociales y aplicaciones móviles enfocadas en la comunicación y marketing. Asimismo, la presente investigación busca determinar el nivel de presencia de las redes sociales en las estrategias de comunicación, promoción y publicidad.

\section{Metodología}

Esta investigación tiene un enfoque cuantitativo. La población comprendida para el estudio fueron las MIPES de la ciudad de Cuenca (Ecuador). Para el efecto se implementó una encuesta con 28 preguntas, la misma que fue dirigida a los encargados del manejo de las TIC, o las personas responsables de la comunicación de marketing de las MIPES.

Este instrumento sirvió para obtener la información de 133 Microempresas y 42 Pequeñas empresas. La investigación abarca a todas las áreas de economía de las MIPES, a través de una estratificación a conveniencia.

Para determinar el tamaño de la muestra se calculó con un nivel de confianza del $95 \%$ para las dos categorías de empresa y un margen de error de $+/-5 \%$ para la Microempresa y $+/-6 \%$ para la Pequeña empresa. Fue utilizado para el procesamiento de datos el programa estadístico IBM SPSS® (Statistical Package for the Social Sciences). El análisis de los resultados tiene una base descriptiva; mientras que los constructos de medición están validados mediante el uso del Alfa de Cronbach resultante de 0,730. Siguiendo a Peterson como se citó en Uribe, Rialp \& Llonch (2013, p. 215) "un valor menor que 0.7 demuestra una baja 
consistencia interna de la escala" por lo que los resultados obtenidos son satisfactorios.

Para determinar el nivel de uso se realizó mediante un escala ordinal y de intervalos que según Romero, Rébori y Camino en la escala "ordinal, se agrega la noción de orden (las categorías poseen un orden que conlleva a la posibilidad de ordenar los datos) y en el intervalar, se agrega la posibilidad de cuantificar distancias entre diferentes categorías o valores de la variable" (2010, p.19). En concordancia con este planteamiento, se determinaron cinco niveles de uso; se asignó un nivel de 0 a 20 como muy bajo, de 21 a 40 nivel bajo, de 41 a 60 como nivel medio, de 61 a 80 nivel alto y de 81 a 100 como un nivel muy alto.

\section{Resultados}

Los resultados de la investigación, una vez realizado el respectivo proceso para la obtención de información, se presentan en dos partes. La primera, correspondiente a las microempresas y la segunda, una comparación entre microempresas y pequeñas empresas. A continuación en el gráfico 1 se presentan los resultados del nivel de uso de las redes sociales y aplicaciones móviles en la microempresa en comparación con la pequeña empresa.

\section{Gráfico 1. Nivel de uso de las redes sociales y aplicaciones móviles}

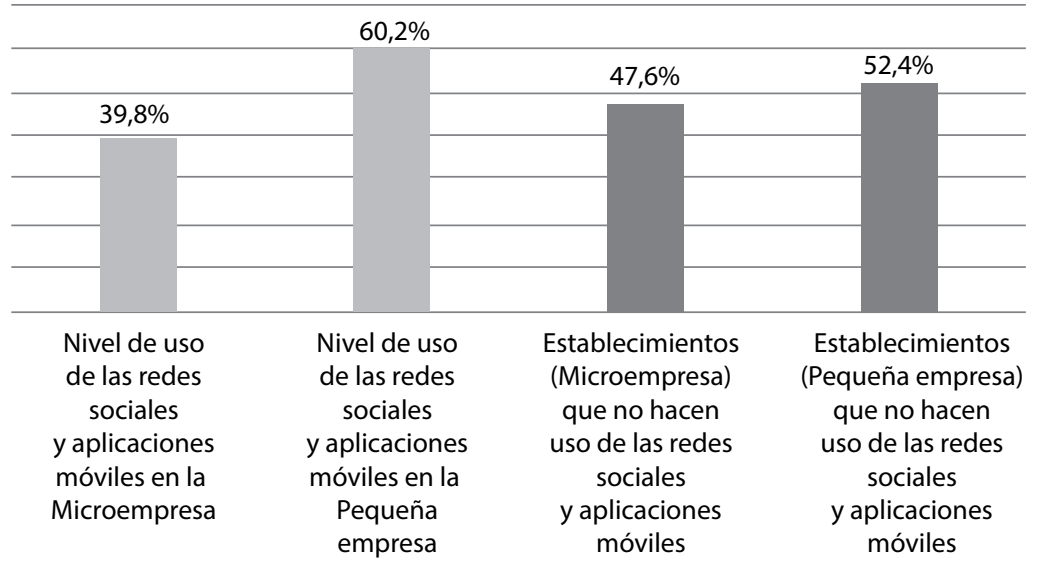




\subsection{Estudio de la microempresa}

Ámbito de cobertura

En las Microempresas, el $83 \%$ de estos establecimientos tienen acceso internet, de los cuales un $70 \%$ señala que la calidad del servicio de internet está entre buena y muy buena. La conexión a la red tiene mayor acogida a través computadoras portátiles y smartphones. En la Microempresa, solo el 39,8\% tiene una cuenta corporativa en redes sociales, en el siguiente gráfico (2) se observan las diferentes redes sociales utilizadas, liderando Facebook con una amplia mayoría de 58\%.

Gráfico 2. Redes sociales y aplicaciones móviles

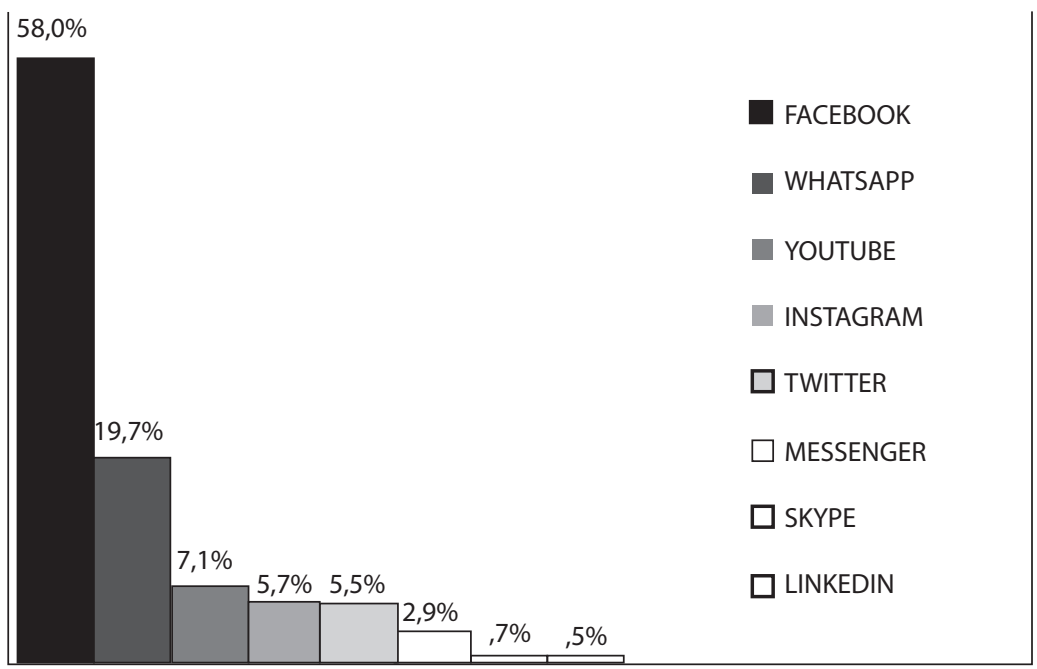

El 60,2\% de establecimientos no hacen uso de las redes sociales. Tras evaluar las razones aducidas, se han tipificado los siguientes aspectos, los mismos que se ilustran en el gráfico 3, demostrando que las principales razones son por: a) Falta de conocimiento en el uso y administración, b) No lo considera necesario, c) Falta de confianza y credibilidad. 
Gráfico 3. Microempresas y las redes sociales

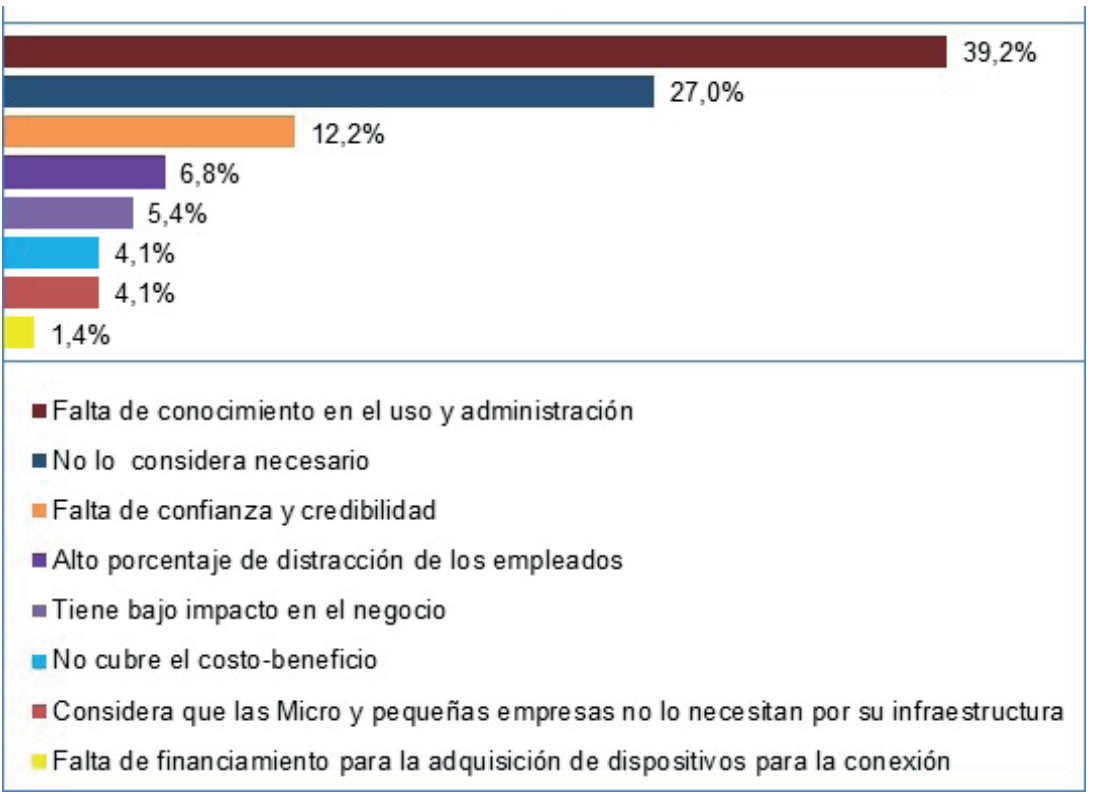

Ámbito de uso y administración

Los resultados proporcionados en este ámbito son de los establecimientos que hacen uso de las redes sociales a través de una o más cuentas corporativas. En concordancia con esto, se demuestra que el 97\% de estos establecimientos utilizan las redes sociales de forma diaria para actividades de comunicación. El 37\% publica y promociona de forma mensual en estos medios, mientras que los demás establecimientos lo hacen en diferentes fechas y ocasiones del año.

Para la gestión de estas actividades, el 38\% de estos establecimientos están conectados a estas redes más de tres horas al día, un 30\% está entre 1 y 3 horas al día y los demás lo consideran menos de una hora al día. En este sentido los usuarios manifestaron mayoritariamente tener un nivel de confianza y credibilidad entre medio y alto en las redes sociales para actividades de comunicación y marketing; los mismos que son utilizados para la consecución de los diferentes fines organizacionales. Ante esto en la tabla 2 se ilustra la relación entre el uso y los objetivos que buscan las organizaciones a través de las redes sociales. 
Tabla 2. Redes sociales y aplicaciones móviles

\begin{tabular}{|c|c|c|c|c|c|}
\hline \multicolumn{6}{|c|}{$\begin{array}{l}\text { Nivel de distribución de las redes sociales } \\
\text { y aplicaciones móviles acorde a los objetivos empresariales }\end{array}$} \\
\hline \multicolumn{3}{|c|}{ Brindar información } & \multicolumn{3}{|c|}{ Mejorar la imagen de la empresa } \\
\hline & Facebook & $74 \%$ & & Facebook & $64 \%$ \\
\hline & Twitter & $11 \%$ & & Twitter & $20 \%$ \\
\hline (0) & Instagram & $9 \%$ & & Instagram & $16 \%$ \\
\hline & WhatsApp & $4 \%$ & \multicolumn{3}{|c|}{ Promociones y acciones publicitarias } \\
\hline & Messenger & $4 \%$ & & Facebook & $90 \%$ \\
\hline \multicolumn{3}{|c|}{ Comunicación con todos los stakeholders } & & Instagram & $10 \%$ \\
\hline & WhatsApp & $81 \%$ & \multicolumn{3}{|c|}{ Para seguimiento post-venta } \\
\hline & Facebook & $11 \%$ & & Facebook & $55 \%$ \\
\hline & Messenger & $6 \%$ & & WhatsApp & $39 \%$ \\
\hline Q & Skype & $2 \%$ & & Messenger & $6 \%$ \\
\hline
\end{tabular}

Ante lo expuesto en la tabla 2, se recalca que existen otros objetivos de uso como: i) observar a la competencia; ii) formación; iii) captar talentos; iv) recoger opiniones y sugerencias; sin embargo para estos objetivos, se hace uso en menor medida las redes sociales y aplicaciones móviles. En este mismo apartado es preciso reiterar que el $83 \%$ de los establecimientos manifiesta como indispensable el uso del chat para envio de archivos, tanto de datos como de voz, actividad que se realiza con todos los stakeholders, destacándose la interacción con el cliente (CRM). Con el interés de conocer la efectividad de la comunicación y el marketing a través de las redes sociales, se realizó una comparación con los medios convencionales, en donde el 79\% de los establecimien- 
tos que hacen uso de las redes sociales y aplicaciones móviles manifestaron que las acciones de comunicación son más efectivas en estos medios, mientras que para acciones de mercadotecnia, el $73 \%$ de encuestados afirman que son efectivos las redes sociales para esta actividad; la diferencia proporcional a cada acción respectivamente indicaron que son efectivos los medios convencionales para estas acciones (comunicación y mercadotecnia).

Por otro lado, en el ámbito administrativo, el 49\% de los directivos o dueños realizan un análisis previo a la implementación de las redes sociales, con el objetivo de identificar los riesgos y beneficios proporcionados por estos medios para los establecimientos, de los cuales el $45 \%$ asegura contar con un community manager, persona que se encarga de administrar el material dirigido a la red. Para este efecto solo el 21\% tiene un plan de capacitación continua para el talento humano de esta área.

En este escenario el $40 \%$ de establecimientos realiza un seguimiento del impacto como estadísticas, conteo de likes, administración de comentarios de las actividades realizadas en la red, el 36\% utiliza Fanpage para acciones de marketing y el $40 \%$ mide el retorno de la inversión de las acciones publicitarias realizadas en las redes sociales.

En aspectos de prevención, tan solo el 11\% tiene un manual o plan para evitar fuga de información a través de estos medios, por lo tanto hay una debilidad bastante evidente en temas de prevención. Por otra parte en aspectos de percepción el $93 \%$ piensa que puede llegar directamente al consumidor final con sus productos/servicios y el $32 \%$ piensa que puede aparecer en las tiendas online con la ayuda de las redes sociales.

En este apartado las Microempresas revelan las ventajas obtenidas por el uso y administración de las redes sociales y aplicaciones móviles en la gestión empresarial en el campo comunicación y de mercadotecnia, las mismas que se observan en el gráfico 4 , resaltando la rapidez en comunicación y difusión de información en $22 \%$.

En este mismo aspecto, la información obtenida a través de estos medios se asegura útil para la toma decisiones, las mismas que contribuyen a mejorar aspectos que carecen de una gestión efectiva o situaciones que desde el punto de vista del cliente no llegan a satisfacer las expectativas esperadas. 


\section{Gráfico 4. Uso y administración de redes sociales y aplicaciones móviles}

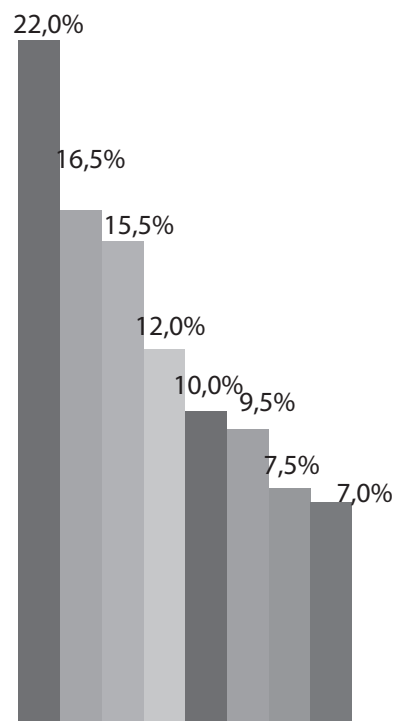

Mayor rapidez en comunicación y difusión de información

Costos bajos de comunicación

Costos bajos en promoción y publicidad

Mayor eficiencia en el servicio

Ser una empresa más competitiva

Aumentar las ventas

Mayor productividad de los colaboradores

Fidelizar al cliente

Por su parte, el 51\% asegura que las redes ayudan a mejorar el servicio, el $31 \%$ opina que mejora la imagen corporativa y el resto (18\%) mejora el producto. Asimismo los encuestados consideran que como se obtienen ventajas por el uso y administración de las redes sociales y aplicaciones móviles, también existen desventajas: El 55\% de los dueños de establecimientos que hacen uso de estos medios manifiestan que es la distracción de los colaboradores, un 26\% piensan que le inspira desconfianza al cliente, mientras que el restante manifiesta que no confían en estos medios.

\subsection{Microempresas vs Pequeña Empresa}

La Pequeña Empresa tiene similitudes muy cercanas con la Microempresa en el uso de las redes sociales y aplicaciones móviles en los campos de comunicación y mercadotecnia, destacando que el tamaño de los establecimientos no influye en mayor proporción en estas áreas de investigación; salvo en algunos aspectos específicos difieren, pero en ninguno de los ámbitos es mayor al 10\%. A conti- 
nuación se puntualizan estas diferencias existentes en los ámbitos de cobertura, uso y administración:

- En el ámbito de cobertura, el 9,9\% más de establecimientos de la pequeña empresa tiene acceso a internet en relación con la microempresa. La acogida por las redes sociales, es mayor en 7,8\% en correspondencia con la microempresa. No obstante existe un nivel bajo en la microempresa y medio en la pequeña empresa en adopción de redes sociales.

- En el ámbito de uso, se ve una disminución en la pequeña empresa de 7,5\% en la utilización diaria de las redes sociales y aplicaciones móviles para actividades de comunicación. Sin embargo, la administración es mayor en la pequeña empresa. Una de las razones es que existe más talento humano contratado para esta área. En relación con la Microempresa difieren en 10 puntos porcentuales, lo que repercute en un ligero aumento en la administración de estos medios, reflejando una mejor gestión de herramientas y páginas como Fanpages. Además se observa un ligero incremento en el manejo de análisis estadísticos, monitoreo y administración de comentarios por acciones de comunicación y mercadotecnia realizados en las redes sociales.

\section{Conclusiones}

La Microempresa y la Pequeña Empresa (MIPES) de Cuenca, si bien es cierto son negocios representativos en la ciudad, alrededor del $90 \%$ de estos establecimientos tienen acceso a internet y dispositivos tecnológicos para sus operaciones. No obstante, según los resultados obtenidos, el nivel de adopción de las redes sociales de acuerdo a la escala establecida es bajo para la microempresa y medio para la pequeña empresa. Las principales razones por las cuales los establecimientos no utilizan estos medios es por: a) Falta desconocimiento en el uso y administración, b) No lo consideran necesario, y c) Falta de confianza y credibilidad entre otros aspectos considerados en menor proporción.

Los establecimientos que hacen uso de las redes sociales y aplicaciones móviles han conseguido, en términos generales, que la relación entre el uso y objetivos propuestos como: comunicación, publicidad, promoción, mejora de la imagen de la empresa, entre otros fines; se logren alcanzar por las organizaciones, resaltando beneficios encontra- 
dos como: Mayor rapidez en la comunicación y difusión de información, mayor eficiencia en el servicio, costos más bajos en comunicación, promoción y publicidad. En cuanto a las acciones publicitarias, el 73\% de estos establecimientos indica que son efectivas las redes sociales para estos fines. Además, en este aspecto, se demuestra que el 90\% de estas actividades se realizan en Facebook, red social líder en audiencia a escala local, nacional e internacional. Sin embargo, se puede concluir en esta investigación que las redes sociales no son explotadas a toda su potencialidad por los establecimientos que hacen uso de estos medios, debido a que únicamente el $40 \%$ de estos establecimientos realiza un seguimiento de impacto como estadísticas, administración y monitoreo de comentarios, entre otras actividades de auditoría y análisis de las redes sociales. Como futuras líneas de investigación se recomienda plantear un nuevo estudio de la comunicación del marketing interno, tema que aportaría para hacer un análisis comparativo entre los dos aspectos.

\section{Referencias}

Alcazar, J. (01 de febreo de 2016). Estadísticas Facebook Ecuador. Obtenido de Formacón Gerencial: (https://goo.gl/gszxXN)

Anzola, S. (2010). Administración de pequeñas empresas. México: McGraw-Hill/Interamerivada.

Barker, M., Barker, D., Nicholas, B., \& Niher., K. (2015). Marketing para medios sociales. Mexico: Cengage learning.

Bigné, E., Küster, I., \& Hernández, A. (2013). Las redes sociales virtuales y las marcas: Influencia del intercambiode experiencias eC2C sobre la actitud de los usuarios hacia la marca[The social networks sites and the brands. Influence of eC2C exchange experience on brand users' Attitudes. Revista Española de investigación En Marketing ESIC, 17(2). 7-27. doi:https://doi.org/10.1016/S11381442(14)60022-X.

Carballar, J. (2012). Social Media marketing personal y profesional. México: Alfaomega.

Casaló, L., Flavián, C., \& Guinalíu, M. (2012). Redes sociales virtuales desarrolladas por organizaciones empresariales: antecedentes de la intención de participación del consumidor[Firm-hosted online social networks: Antecedents of consumer intention to participate]. Cuadernos de Economía y Dirección de la Empresa, 15(1). 42-51. (http://bibliotecavirtual.ups.edu.ec:2353/10.1016/j. cede.2011.06.003).

Elizalde, M., \& Morocho, M. (2014). Análisis para la elaboración de una guia para el manejo estrátegico de Facebook y Twitter para la pequeña empresa cuencana. (tesis de pregrado).Universidad del Azuay, Cuenca, Ecuador.

Estrade, J., Soro, D., \& Hernández, Á. (2013). Marketing Digital. Madrid: Ediciones Anaya. 
Garrido, F. (2004). Comunicación Estrategica. España: Ediciones Gestión 2000.

Gómez, Á., \& Suárez, C. (2012). Sistemas de información. Madrid: RA-MA Editorial. González, Ó. (2014). Comercio Electrónico 2.0. Madrid: Ediciones Anaya.

Hoyos, A., \& Lasso, M. (2017). Pymes como modelo económico en la creación de estrategias de comunicación [SMEs as an economic model in the creation of communication strategies]. Retos, 7(13), 59-74. doi:http://dx.doi.org/10.17163/ret. n13.2017.04.

Hütt Herrera, H. (2012). Las redes sociales: una nueva herramienta de difusión. Reflexiones, 91(2),121-128. (https://goo.gl/Ofx03c).

INEC. (2014). Directorio de empresas y establecimientos. Obtenido de (https://goo.gl/ $\mathrm{n} 4 \mathrm{H} 1 \mathrm{IC})$

Jiménez, M. (2011). La gestion Informatica de la empresa. Madrid: RA-MA Editorial.

Lamb, C., Hair, J., \& McDaniel, C. (2014). MKTG Marketing. méxico: Cengage Learning.

Laudon, J., \& Laudon, K. y. (2012). Sistemas de Información Gerencial. México: Pearson Educación.

Laudon, K. C., \& Traver, C. G. (2014). E-comerce 2013. Mexico: Pearson Educación.

León, J. d. (2012). Guía de usos y estilos en las redes sociales. Obtenido de (https://goo. $\mathrm{gl} / \mathrm{utP} 6 \mathrm{Ld})$

Liberona, D., \& Ruiz, M. (21 de 09 de 2013). Sience Direct. Obtenido de (http://bibliotecavirtual.ups.edu.ec:2229/science/article/pii/S0123592313000041)

Lozares, C. (1996). La teoría de redes sociales. Universitat Autónoma de Barcelona. Departamento de Sociología, (48), 103-126.(file://C:/Users/usuario/Downloads/ 25386-58835-1-PB\%20(2).pdf).

Martos, A. (2010). Redes Sociales. Madrid: Ediciones Anaya.

Medina, A., \& Montjoy, R. (2015). El marketing directo en las redes sociales y su influencia en el campo empresarial. (Tesis de pregrado). Universidad Técnica de Machala, Machala, Ecuador.

Mejía, J. (2013). La Guía del Community Manager. Madrid: Ediciones Anaya.

Moyano, J., Bruque, S., \& Eisenberg, J. (2007). La influencia de las redes socialesen la adaptación de los trabajadores en el cambio tecnólogico. Cuademos de Economía y Dirección de la Empresa, (31), 147-170. (http://bibliotecavirtual.ups.edu. ec:2211/ehost/pdfviewer/pdfviewer?sid=b8955c12-5861-4575-91a9-8e3c5c$44858 \mathrm{f} \% 40$ sessionmgr4010\&vid=4\&hid=4106).

Romero, C., Alrcón, M., \& Gómez, M. (2011). Adopción de redes sociales virtuales: ampliación del modelo de aceptación tecnológica integrando confianza y riesgo percibido[Adoption of social networking sites: extending the technology acceptance model integrating trust and perceived risk]. Cuadernos de Economía y Dirección de la Empresa, 14(3). 194-205. (http://bibliotecavirtual.ups.edu. ec:2353/10.1016/j.cede.2010.12.003).

Romero, M., Rébori, A., \& Camino, M. (2010). Un índice para "medir" el nivel de innovación tecnologica en las empresas. RAI (Revista de Administração e Inovação), 7(1).3-20. (https://goo.gl/0CDoim).

Rosero, C., \& Montalvo, H. (2015). La disonancia cognitiva como factor motivador en las decisiones del consumidor: caso Smartphone [The cognitive dissonance 
as a motivating factor in consumer decisions: smartphones case. Retos, 5(10), 177-192. doi: http://dx.doi.org/10.17163/ret.n10.

Stumpo, M. D. (2002). Análisis de la política de fomento a las pequeñas y medianas empresas en Chile. Santiago de Chile: Impreso en Naciones Unidas, Santiago De Chile.

Tobar, L. (2015). Análisis competitivo de las pequeñas y medianas empresas en Cuenca Ecuador [Competitive analysis of small and medium enterprises in Cuenca, Ecuador]. Revista Internacional Administración Ë Finanzas, 8(3), 79-92. (https:// goo.gl/MPS80p).

Ureña, G., \& Valenzuela, J. (2011). Contactos de redes sociales en línea como repositorios de información. RUSC, 8(1). 128-141. (https://search.proquest.com/ docview/1018856266? accountid=32861).

Uribe, F., Rialp, J., \& Llonch, J. (2013). El uso de las redes sociales digitales como herramienta de marketing en el desempeño empresarial. Universitat Autònoma de Barcelona, 205-231. (http://bibliotecavirtual.ups.edu.ec:2211/ehost/pdfviewer/ pdfviewer?vid=4\&sid=0faeb380-aceb-4fb9-86c7-03f2c09af16d\%40sessionmgr4009\& hid=4101).

Valls, M. (2016). Redes sociales herramienta de gestión empresarial. Buenos Aires: Ugerman editor. 